\title{
Efficient luminescence from easily prepared three-coordinate copper(I) arylamidophosphines $\dagger$
}

\author{
Kenneth J. Lotito $\ddagger$ and Jonas C. Peters* \\ Received 13th January 2010, Accepted 30th March 2010 \\ First published as an Advance Article on the web 14th April 2010 \\ DOI: $10.1039 / \mathbf{c 0 0 0 8 1 8 d}$
}

A series of brightly luminescent, three-coordinate copper(I) arylamidophosphine complexes have been prepared from readily available precursors in high yield. Emission maxima span $102 \mathrm{~nm}$ in the visible spectrum from 461 (blue) to $563 \mathrm{~nm}$ (yellow) while photoluminescence quantum yields range from 0.11 to 0.24 in fluid solution at room temperature.

Synthetic simplicity is a desirable property for luminescent molecular systems as it can facilitate the rapid expansion and tuning of photophysical properties to targeted functions including biological imaging, ${ }^{1}$ photochemical catalysis, ${ }^{2}$ light-driven fuel production, ${ }^{3}$ and electroluminescent devices. ${ }^{4}$ Indeed, their comparative ease of synthesis has in part driven the widespread success enjoyed by iridium cyclometalates in roles ranging from dopants in OLED displays to sensitizers in photochemical hydrogen production. ${ }^{5,6}$

Copper-based luminophores ${ }^{7}$ have been investigated as an inexpensive alternative to the more ubiquitous noble metal emitters and recently our group introduced copper(I) amidophosphine complexes as a class of highly luminescent compounds. To date we have focused on binuclear $^{8}$ and mononuclear ${ }^{9}$ copper(I) complexes that feature tridentate and bidentate arylamidophosphine ligands, respectively. While these compounds exhibit quantum efficiencies as high as $70 \%$ with microsecond luminescence lifetimes, their synthetic versatility hinges on the steps required for the synthesis of the chelating ligands themselves. These steps include (i) catalytic aryl amination to assemble the ligand backbone and (ii) use of lithium reagents and/or strong phosphide nucleophiles to install the phosphine unit.

We now report that highly emissive, mononuclear, threecoordinate copper(I) arylamidophosphines can be quickly assembled from common, commercially available reagents such as diphenylamine and triphenylphosphine. While their quantum efficiencies are not in general as high as those complexes highlighted above, ${ }^{8,9}$ they are favorable when compared to other classes of copper emitters. ${ }^{7}$ Moreover, their ease of structural variation makes this class of complexes a convenient family for further expansion and study.

Department of Chemistry, Massachusetts Institute of Technology, Cambridge, MA 02139, USA

$\dagger$ Electronic supplementary information (ESI) available: Synthetic procedures and characterization, absorption spectrum of $\mathbf{6}$, excitation and emission spectra, electrochemical data, and solid state structures. CCDC 766167 (1), 766168 (2), 766169 (3), 766170 (7). For ESI and crystallographic data in CIF or other electronic format see DOI: $10.1039 / \mathrm{c} 000818 \mathrm{~d}$

\pm Current address: Division of Chemistry and Chemical Engineering, California Institute of Technology, 101-20 Pasadena, CA 91125, USA.

E-mail: jpeters@caltech.edu; Fax: + 1626395 6948; Tel: + 16263954036.

Compounds $\mathbf{1 - 5}$, and $\mathbf{7}$ are prepared by treating a suspension of $\mathrm{CuBr} \cdot \mathrm{SMe}_{2}$ in benzene with two equivalents of triarylphosphine followed by salt metathesis with the corresponding lithium amide. Compound $\mathbf{6}$ is prepared in an analogous fashion employing a single equivalent of the chelating ligand DPEphos (DPEphos = bis-[2-(diphenylphosphino)phenyl]ether). 1-6 are obtained as diamagnetic, bright yellow-green solids while 7 is obtained as a diamagnetic pale yellow solid whose luminescence is only visible upon ultraviolet irradiation. Analytically pure material of $\mathbf{1 - 7}$ is obtained in good to excellent yield (64-91\%) following filtration to remove lithium bromide and recrystallization from a mixture of $n$-pentane and benzene (Fig. 1). Alternatively, 1 can be prepared in substantially lower yield $(19 \%)$ by treating a benzene solution of mesitylcopper(I) with triphenylphosphine and diphenylamine. In all cases, crystals suitable for single crystal X-ray diffraction are obtained by layering a benzene solution of the copper complex with $n$-pentane.

All of the reported complexes are thermally stable but are highly sensitive to adventitious oxygen and water and discolor rapidly in aerated solution giving insoluble brown solids with concomitant loss of luminescence. The complexes are relatively robust in the solid-state, however, with powdered materials retaining both their color and bright luminescence after several weeks in the open atmosphere. Compounds 1-5 and 7, which are prepared with monodentate phosphine ligands, exhibit some decomposition in solution upon dilution to micromolar concentrations as determined by UV-vis spectroscopy. Excitation spectra, however, can be obtained for each compound and provide an indication of their respective absorption profiles (see $\mathrm{ESI} \dagger$ ). In contrast, 6, which is prepared with the chelating phosphine DPEphos, is more robust at low concentrations, suggesting that phosphine dissociation may be problematic for $\mathbf{1 - 5}$ and $\mathbf{7}$ at low concentrations.

The solid-state structures of $\mathbf{1 - 3}$ and $\mathbf{7}$ were determined by single-crystal X-ray diffraction and revealed monomeric complexes in which the amide ligand adopts a monodentate coordination mode (Fig. 2). Well defined examples of monomeric copper complexes with terminal amide ligands are rare

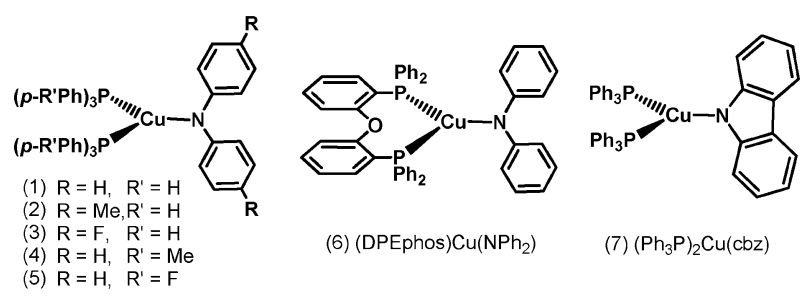

Fig. 1 Schematic representation of complexes 1-7. 


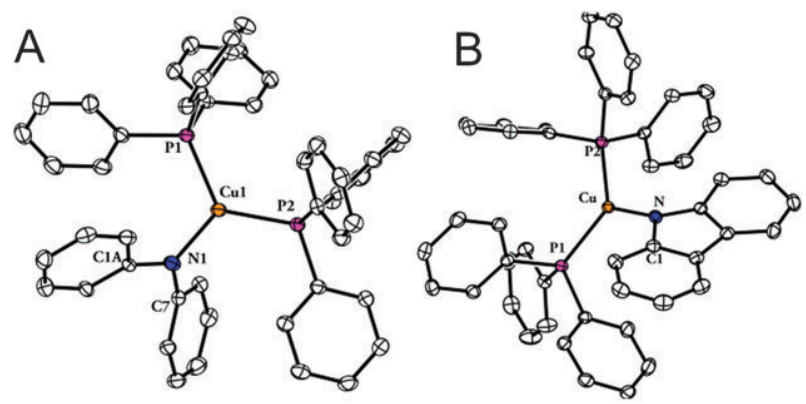

Fig. 2 (A) Displacement ellipsoid representation of 1. (B) Displacement ellipsoid representation of 7 . Thermal ellipsoids at $50 \%$ probability. Hydrogen atoms, solvent, and the minor component of the disorder omitted for clarity.

and were first reported in the past few years. ${ }^{10,11}$ Complex 7 is, to the best of our knowledge, the first reported carbazolate complex of copper.

Each structurally characterized complex exhibits a threecoordinate metal center with a nearly ideal trigonal planar geometry; $\mathbf{1 - 3}$ and $\mathbf{7}$ are rigorously planar as indicated by the angles about copper which sum to $360.00^{\circ}$ within experimental error (see ESI $\dagger$ for structural data) and in all cases the $\mathrm{P}-\mathrm{Cu}-\mathrm{P}$ and $\mathrm{N}-\mathrm{Cu}-\mathrm{P}$ bond angles are within $10^{\circ}$ of the ideal value of $120^{\circ}$. The amido ligand exhibits a large $\mathrm{C}-\mathrm{N}-\mathrm{Cu}-\mathrm{P}$ dihedral angle suggesting little to no contribution of an $\mathrm{N}(\pi) \rightarrow \mathrm{Cu}(4 \mathrm{p})$ interaction to the copper-amide bonding. The $\mathrm{Cu}-\mathrm{N}$ internuclear distances range from 1.9363(17) (2) to 1.9602(16) $\AA$ (1), which fall between those reported for other structurally characterized examples. ${ }^{10}$ Variation of the substituent in the amido ligand in the series 1-3 has no apparent structural consequence.

Complexes 1-7 exhibit pseudo- $C_{2}$ symmetry in the solidstate, with the two-fold axis coincident with the $\mathrm{Cu}-\mathrm{N}$ vector. $\mathrm{C}_{2}$-symmetry is preserved in benzene solution at room temperature according to ${ }^{1} \mathrm{H}$ and ${ }^{31} \mathrm{P}$ NMR measurements that show a single phosphorous resonance and equivalent protons on the amide ligands, where expected (see ESI $\dagger$ ).

The electrochemical properties of each complex were investigated by cyclic voltammetry. Compounds 1 and 3-7 exhibit irreversible oxidation waves around $+600 \mathrm{mV}$ versus the ferrocene/ferrocenium couple in THF. In contrast, complex 2 exhibits a fully reversible oxidation at $+420 \mathrm{mV}$. (See ESI $\dagger$ for detailed electrochemical results.)

Each copper complex is brightly luminescent in both the solid-state and in solution at room temperature. The parent diphenylamide complex (1) exhibits a featureless green emission band centered at $521 \mathrm{~nm}$ in methylcyclohexane $(\mathrm{MeCy})$ at room temperature (Fig. 3 and 4). Substitution with fluorine at the para position of the amide phenyl rings (3) results in a negligible bathochromic shift in $\lambda_{\mathrm{em}}$ while functionalization with methyl groups (2) shifts the emission by $25 \mathrm{~nm}$ to longer wavelength. In contrast to the diphenylamido complexes, which tend towards yellow-green emission, copper carbazolate 7 exhibits cyan emission centered at $461 \mathrm{~nm}$ and demonstrates that the photophysical properties of this series of compounds are highly dependent on the identity of the amide ligand.

Variation of the ancillary phosphine also has an impact on the emission wavelength. Substituting triphenylphosphine for

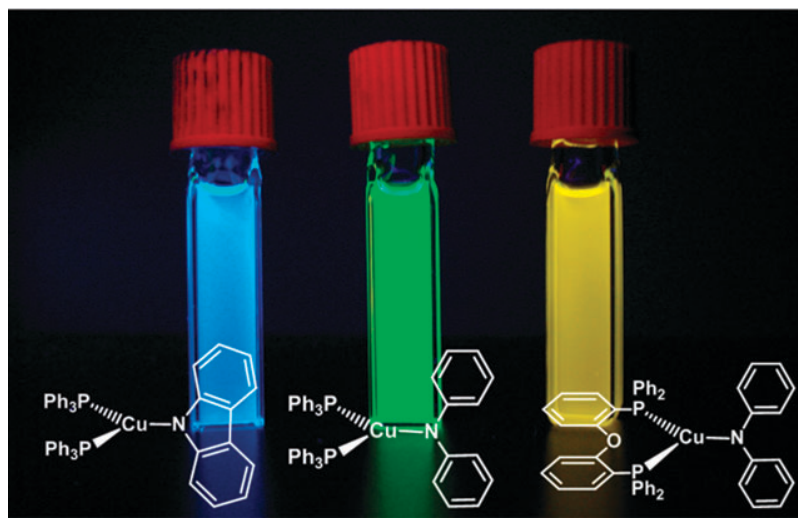

Fig. 3 Photoluminescence from compounds 1, 6, and 7 in $\mathrm{MeCy}$ upon long-wave UV irradiation.

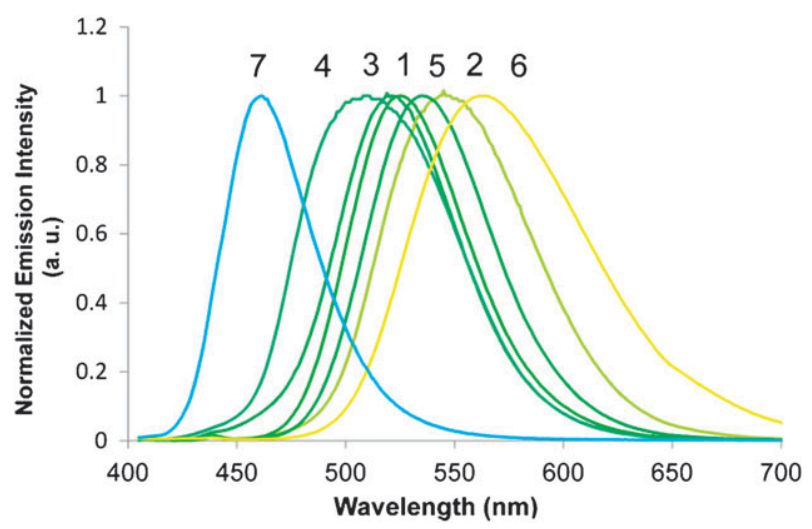

Fig. 4 Photoluminescence spectra of 1-7 in $\mathrm{MeCy}$ at room temperature. $\lambda_{\mathrm{ex}}=390 \mathrm{~nm}$.

the more electron-releasing tri-p-tolylphosphine (4) results in a modest $12 \mathrm{~nm}$ blue shift in the emission while employing the relatively electron withdrawing $\mathrm{P}(p-\mathrm{FPh})_{3}$ ligand (5) shifts the emission wavelength $14 \mathrm{~nm}$ to the red. While these results suggest that the room temperature emission maximum may be rationally tuned by judicious choice of phosphine electronic properties, substitution of triphenylphosphine for the electronically similar DPEphos (6) results in a substantial red shift, giving a broad, yellow emission centered at $563 \mathrm{~nm}$.

Solution quantum yields were determined by comparison of the integrated emission intensity to a standard sample of perylene and excited-state lifetimes were determined by timeresolved emission spectroscopy (Table 1). 1-7 are comparably efficient emitters, with $\Phi_{\mathrm{PL}}$ varying over the relatively narrow range $0.11-0.24$. Each complex exhibits a monoexponential luminescence decay profile in solution with lifetimes on the microsecond timescale suggesting the involvement of a triplet excited state. The lifetimes for the isostructural series 1-3 and $\mathbf{1}, \mathbf{4}$, and $\mathbf{5}$ show little alteration upon para-substitution of the amide and phosphine ligands. Exchange of the diphenylamide for the more rigid carbazolate ligand, however, results in a four-fold enhancement of the lifetime. While not as bright as the amidophosphine complexes supported by chelating ligands that we have reported previously, ${ }^{8,9}$ complexes 1-7 do represent a substantial improvement over copper bis-diimine complexes $\left(\Phi_{\mathrm{PL}} \approx 0.01\right)$ and compare favorably to heteroleptic 
Table 1 Photophysical parameters of copper complexes at room temperature in $\mathrm{MeCy}$

\begin{tabular}{llll}
\hline Complex & $\lambda_{\mathrm{em}} / \mathrm{nm}$ & $\Phi_{\mathrm{PL}}{ }^{a, b}$ & $\tau / \mu \mathrm{s}^{c}$ \\
\hline $\mathbf{1}$ & 521 & 0.23 & $3.17(5)$ \\
$\mathbf{2}$ & 546 & 0.22 & $3.1(2)$ \\
$\mathbf{3}$ & 525 & 0.13 & $2.5(1)$ \\
$\mathbf{4}$ & 509 & 0.13 & $2.5(1)$ \\
$\mathbf{5}^{d}$ & 535 & 0.11 & $2.71(2)$ \\
$\mathbf{6}$ & 563 & 0.18 & $1.70(1)$ \\
$\mathbf{7}$ & 461 & 0.24 & $11.7(6)$
\end{tabular}

${ }^{a}$ Uncertainty in quantum yield measurements is estimated to be \pm 0.05 . $b \lambda_{\mathrm{ex}}=390 \mathrm{~nm} . c \lambda_{\mathrm{ex}}=337 \mathrm{~nm} .{ }^{d}$ In $\mathrm{C}_{5} \mathrm{H}_{10}$.
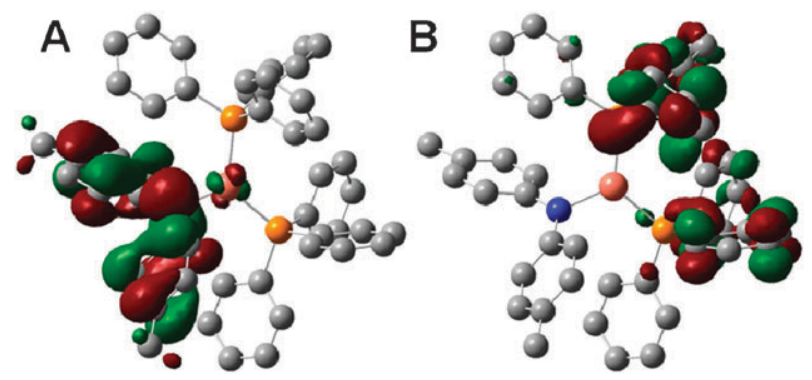

Fig. 5 (A) HOMO of $\mathbf{2}$ as calculated by DFT. Energy = $-84.3 \mathrm{kcal} \mathrm{mol}^{-1}$. (B) LUMO of $\mathbf{2}$ as calculated by DFT. Energy $=$ $-23.4 \mathrm{kcal} \mathrm{mol}^{-1}$. Isovalue $=0.03$. Hydrogen atoms omitted for clarity.

phosphinodiimine complexes $\left(\Phi_{\mathrm{PL}} \leq 0.16\right)$ that have been the most extensively studied monomeric copper emitters. ${ }^{7}$

The electronic structure of $\mathbf{2}$ was explored using Density Functional Theory (DFT) calculations at the B3LYP/ $6-31+\mathrm{G}^{*}$ level of theory. The computed highest occupied molecular orbital (HOMO) is localized primarily on the diphenylamide ligand with substantial nitrogen $\mathrm{p} \pi$ and $\mathrm{C}-\mathrm{C}$ $\pi$ character (Fig. 5). There is a small contribution from a $\mathrm{Cu}$ $\mathrm{d}$-orbital that is $\pi$-antibonding with respect to the amide p-orbital. The HOMO is nearly identical to those calculated for a related class of complexes ${ }^{9}$ and indicates that tuning of the ground state energy is possible through ortho- or parasubstitution of the amide phenyl rings. The calculated lowest unoccupied molecular orbital (LUMO) is localized on the triphenylphosphine ligands and has $\mathrm{C}-\mathrm{C} \pi^{*}$ character. While these computational results imply a ligand-to-ligand-chargetransfer (LLCT) excitation, computational and experimental studies on related systems suggest that amide ligand-centered (LC) excited states may also play an important role. ${ }^{10,12}$ Further studies aimed at understanding the photophysics and electronic structure of these luminescent copper(I) amidophosphines are underway for comparison to a recent detailed photophysical study on the dicopperphosphinoamide complex $\left[\mathrm{Cu}\left(\mathrm{PNP}-{ }^{t} \mathrm{Bu}\right)\right]_{2}\left(\mathrm{PNP}^{-}{ }^{t} \mathrm{Bu}=\operatorname{bis}[(2\right.$-diisopropylphosphino-4tert-butyl)phenyl]amide). ${ }^{13}$

In conclusion, we have described a series of three-coordinate copper(I) diarylamidophosphine complexes that combine efficient luminescence in solution at room temperature, spectroscopic tunability, and ease of synthesis. The ease of synthetic modification afforded by these complexes provides an opportunity to expand the available emission colors and to facilitate a more detailed study of the photophysical states contributing to their luminescence properties.

This work was supported by an NSF Center for Chemical Innovation (CHE-0802907). Luminescence measurements were carried out in the laboratory of T. M. Swager with assistance from T. L. Andrews. K. J. L. gratefully acknowledges a fellowship from the MIT Energy Initiative.

\section{Notes and references}

1 J. Riegler and T. Nann, Anal. Bioanal. Chem., 2004, 379, 913-919.

2 P. Ciesla, P. Mytych and Z. Stasicka, J. Mol. Catal. A: Chem, 2004, 227, 17-33.

3 G. M. Brown, B. S. Brunschwig, C. Creutz, J. F. Endicott and N. Sutin, J. Am. Chem. Soc., 1979, 101, 1298-1300; N. Robertson, ChemSusChem, 2008, 1, 977-979.

4 H. Yersin, Top. Curr. Chem., 2004, 241, 1-26; M. Noto, Y. Goto and M. Era, Chem. Lett., 2003, 32-33.

5 M. A. Baldo, M. E. Thompson and S. R. Forrest, Nature, 2000, 403, 750-753; S. Lamansky, P. Djurovich, D. Murphy, F. AbdelRazzaq, H.-E. Lee, C. Adachi, P. E. Burrows, S. R. Forrest and M. E. Thompson, J. Am. Chem. Soc., 2002, 123, 4304-4312.

6 E. D. Cline, C. M. Kraml, N. Byrne, D. M. Ho, Q. Qin, F. J. Coughlin, S. Bernhard and R. A. Pascal, Inorg. Chem., 2008, 47, 10378-10388; J. I. Goldsmith, W. R. Hudson, M. S. Lowry, T. H. Anderson and S. Bernhard, J. Am. Chem. Soc., 2005, 127, 7502-7510.

7 N. Armaroli, G. Accorsi, F. Cardinali and A. Listorti, Top. Curr. Chem., 2007, 280, 69-115; P. C. Ford, E. Cariati and J. Bourassa, Chem. Rev., 1999, 99, 3625-3627; O. Horvath, Coord. Chem. Rev., 1994, 135/136, 303-32; V. Balzani, A. Juris, M. Venturi, S. Campagna and S. Serroni, Chem. Rev., 1996, 96, 759-833; R. F. Ziolo, S. Lipton and Z. Dori, J. Chem. Soc. D, 1970, 1124-1125; C. Kutal, Coord. Chem. Rev., 1990, 99, 213-252; M. W. Blaskie and D. R. McMillin, Inorg. Chem., 1980, 19, 3519-3522; D. G. Cuttell, S.-M. Kuang, P. E. Fanwick, D. R. McMillin and R. A. Walton, J. Am. Chem. Soc., 2002, 124, 6-7; A. M. James, R. K. Laxman, F. R. Fronczek and A. W. Maverick, Inorg. Chem., 1998, 37, 3785-3791.

8 S. B. Harkins and J. C. Peters, J. Am. Chem. Soc., 2005, 127, 2030-2031.

9 A. J. M. Miller, J. M. Dempsey and J. C. Peters, Inorg. Chem., 2007, 46, 7244-7246.

10 E. D. Blue, A. Davis, D. Conner, T. B. Gunnoe, P. D. Boyle and P. S. White, J. Am. Chem. Soc., 2003, 125, 9435-9441; L. A. Goj, E. D. Blue, C. Munro-Leighton, T. B. Gunnoe and J. L. Petersen, Inorg. Chem., 2005, 44, 8647-8649; P. Reiß and D. Z. Fenske, Z. Anorg. Allg. Chem., 2000, 626, 1317-1331; N. P. Mankad, W. E. Antholine, R. K. Szilagyi and J. C. Peters, J. Am. Chem. Soc., 2009, 131, 3878-3880; M. M. Melzer, S. Mossin, X. Dai, A. M. Bartell, P. Kapoor, K. Meyer and T. H. Warren, Angew. Chem., Int. Ed., 2010, 49, 904-907.

11 Yamamoto and co-workers had previously formulated a series of $\mathrm{Cu}(\mathrm{I})$ bisphosphinoamidates as three-coordinate. No structural data were presented. One of these complexes was observed to be luminescent: T. Yamamoto, Y. Ehara, M. Kubota and A. Yamamoto, Bull. Chem. Soc. Jpn., 1980, 53, 1299-1302.

12 S. B. Harkins, N. P. Mankad, A. J. Miller, R. K. Szilagyi and J. C. Peters, J. Am. Chem. Soc., 2008, 130, 3478-3485.

13 J. C. Deaton, S. C. Switalski, D. Y. Kondakov, R. H. Young, T. D. Pawlik, D. J. Giesen, S. B. Harkins, A. J. M. Miller, S. F. Mickenberg and J. C. Peters, 2010, manuscript submitted for publication. 